INPLASY

PROTOCOL

To cite: Dalawi et al.

Knowledge, Attitude and

Practice Assessment on

COVID-19 infection: A

Systematic Review. Inplasy

protocol 202150039. doi:

10.37766/inplasy2021.5.0039

Received: 11 May 2021

Published: 11 May 2021

Corresponding author:

Mohamad Rodi Isa

rodi@uitm.edu.my

Author Affiliation:

Department of Public Health

Medicine, Faculty of Medicine,

Universiti Teknologi MARA

Sungai Buloh Campus.

Support: MyRA Research

Grant 2020.

Review Stage at time of this submission: Preliminary

searches.

Conflicts of interest:

None declared.

\section{Knowledge, Attitude and Practice Assessment on COVID-19 infection: A Systematic Review}

Dalawi, I1; Isa, MR²; Chen, XW33; Azhar, ZI4; Aimran, AN55.

Review question / Objective: What are the Knowledge, Attitude, and Practice (KAP) assessment tool on COVID-19 infection available in the literature? What is the reliability and validity of the available KAP assessment tools on COVID-19? Condition being studied: We will be studying the assessment tools on Knowledge, attitudes, and practice related to COVID-19 infection throughout the countries.

Information sources: WHO COVID-19, Science Direct, Scopus, PubMed, MEDLINE, and EMBASE databases. Grey Literatures will not be included in the study.

INPLASY registration number: This protocol was registered with the International Platform of Registered Systematic Review and Meta-Analysis Protocols (INPLASY) on 11 May 2021 and was last updated on 11 May 2021 (registration number INPLASY202150039).

\section{INTRODUCTION}

Review question / Objective: What are the Knowledge, Attitude, and Practice (KAP) assessment tool on COVID-19 infection available in the literature? What is the reliability and validity of the available KAP assessment tools on COVID-19?
Rationale: For more than a year, the global has lived with the SARS-COV-2 virus. Yet, this infection is continued to spread globally. While in Malaysia, we could see that one wave after another hit the country with the latest re-surging of cases since early April 202. This has been postulated that could be a sign of pandemic fatigue in the population, which can be defined as 
demotivation to follow the recommended protective behaviors, emerging gradually over time and affecting many emotions, experiences, and perceptions. Furthermore, the uncertainties, fake news, and misunderstanding on the COVID-19 itself would cause a lot of confusion among the public. Thus, this can be directly affecting how people react and behave towards COVID-19, in which any incongruence may lead to the deterioration of the preventive and control behaviors. Consequently, this indicates the need to assess the knowledge, attitude, and practice (KAP) towards COVID-19 and the anticipated challenges for such assessment in the post-COVID-19 era. The KAP survey is very useful as the data generated from the survey can identify the knowledge gaps, cultural beliefs, and behavioral patterns, which can further identify the needs and barriers in implementing certain health interventions. Also, the data will deepen the understanding, attitude, practice, and the factors associated and act as baseline data towards the studied topic and behavior. These will help the policymaker and stakeholders to be able to set a program and decision depending on the priorities. In the case of COVID-19, the effect of the implementation of movement control orders, health education campaigns, and vaccination programs can also be measured using the KAP survey, which can also be done from time to time. Till now, the basic public health intervention such as hand hygiene, social and physical distancing, and wearing masks with or without eye protection remains as the best weapons to combat the disease regardless of the vaccine's arrival. The effectiveness of these measures is very much dependent on the cooperation and compliance of the population. On top of that, following the vaccination program on COVID-19, which has started, the KAP survey can also be one of the approaches to assess the impact from time to time, especially in terms of the factors associated with the acceptability of the vaccines. To support the importance of the KAP survey and the questionnaire used, we need to ensure that the data gained from the KAP questionnaire should be reliable and valid for the usage of the intended population so that it can help to collect a better quality of data with high comparability and credibility of the data. It should be properly developed, validated, and psychometrically sound. To our knowledge, there are no published systematic reviews that focus on the assessment tool of the knowledge, attitude, and practice of COVID-19, similar to our study. Previous systematic reviews may cover the patterns of knowledge, attitude, and practices on the COVID-19 in Eutopia and the associated factors. This review aims to identify, describe and evaluate the KAP assessment tool on COVID-19 available in the literature. A comprehensive review of all questionnaires on the KAP studies on COVID-19 will be collated and confirm the valid and reliable assessment tool for future KAP research in this field.

Condition being studied: We will be studying the assessment tools on Knowledge, attitudes, and practice related to COVID-19 infection throughout the countries.

\section{METHODS}

Search strategy: The search strategy aims to obtain published articles only. The process of searching strategy for identification of studies will include all published studies. It involved a three-stage protocol. The first stage will be followed by analyzing the text words contained in the titles and abstracts and of the index terms used to describe each article. The published articles referring to any article that has been published in peer-reviewed indexed journals published articles will be located for this research using computerbased information search. The established databases to be searched in this study will be WHO COVID-19, Science Direct, Scopus, PubMed, MEDLINE, and EMBASE. Researchers will use search terms such as knowledge, attitude, practice, assessment, COVID-19 infection. In the second stage, a Boolean search will be conducted using the identified keywords of the selected databases. Boolean search will be 
performed on each database using the search term: "COVID-19" AND ("assessment" OR "questionnaire" OR "survey") AND ("knowledge" OR "attitude" OR "practice"). The published literature will be carefully searched for this study. An initial limited search of WHO COVID-19, Science Direct, Scopus, PubMed, MEDLINE, and EMBASE databases will be undertaken. The study will be restricted to research articles and case reports, human study, English language, and articles published from year December 2019 to January 2021. Only cross-sectional studies will be considered. The exclusion criteria are review articles, in vivo or in vitro studies, other languages, non-free full-text articles. In the third stage, the reference lists of key articles will be searched for additional studies. The selection of studies will follow PRISMA guidelines. Only a satisfactory quality of the papers using the STROBE checklist for the cross-sectional studies will be entered for data extraction. Three independent researchers will perform the critical appraisal to assess study quality and minimize bias. Most methods encompass issues such as appropriateness of study design to the research objective. The articles which do not fulfill the and poor-quality studies will be excluded or discussed in detail. Any disagreements between the reviewers will be resolved through discussion and consideration to the fourth reviewer.

Participant or population: The assessment tools used to measure the knowledge, attitudes, and practice of COVID-19 in a cross-sectional study.

Intervention: Not applicable.

Comparator: Not applicable.

Study designs to be included: Cross sectional study.

Eligibility criteria: The inclusion criteria in this study are research articles and case reports, human studies, English language, and articles published from December 2019 to January 2021. The exclusion criteria are review articles, in vivo or in vitro studies, other languages, non-free full-text articles. This systematic review will consider the only cross-sectional study of good quality.

Information sources: WHO COVID-19, Science Direct, Scopus, PubMed, MEDLINE, and EMBASE databases. Grey Literatures will not be included in the study.

Main outcome(s): The outcome measures reported in studies meeting the inclusion criteria will be grouped under the following headings: country, questionnaire items, study population, language, number of items in each domain (knowledge, attitude, and practice), and the validity and reliability of each domain.

\section{Additional outcome(s): Not applicable.}

Data management: All relevant articles will be manually coded in the spreadsheets as described in evidence tables. The electronic spreadsheets will be utilized to import the data into excel form for data analysis.

Quality assessment / Risk of bias analysis: The methodological quality of the studies will be assessed using the Strengthening the Reporting of Observational Studies in Epidemiology (STROBE) for a crosssectional study. Any disagreements that arise between the reviewers will be resolved through discussion and taking consideration to the fourth reviewer.

Strategy of data synthesis: Data extraction will be performed by two independent researchers to establish inter-rater reliability and to avoid data entry errors. Study context factors of published articles will include the information describing the study and its subjects. Reported findings of the remaining studies will be extracted onto a data extraction form. Lists of included studies will be then created. Data extraction will be independently crosschecked.

Subgroup analysis: Not applicable.

Sensitivity analysis: Not applicable. 
Language: English.

Country(ies) involved: Malaysia.

Keywords: knowledge, attitude, practice, assessment, COVID-19 infection.

Dissemination plans: From this systematic review, two papers i.e. the manuscript on the protocol and the findings of the systematic review will be submitted to a leading journal in this field.

Contributions of each author:

Author 1 - Izzaty Dalawi The author conceived the idea, planned and designed the study protocol, wrote the first draft, planned the data extraction and statistical analysis.

Email: izzatydalawi@gmail.com

Author 2 - Mohamad Rodi Isa - The author conceived the idea, planned and designed the study protocol, wrote the first draft, planned the data extraction and statistical analysis.

Email: rodi@uitm.edu.my

Author 3 - Chen Xin Wee - The author conceived the idea, provided critical insights into the study protocol, and provided the feedback for the approval of the manuscript.

Email: drchenxw@uitm.edu.my

Author 4 - Zahir Izuan Azhar - The author also conceived the idea and provided feedback for the approval of the final study protocol and manuscript.

Email: drzahir@uitm.edu.my

Author 5 - Ahmad Nazim Aimran - The author provided critical insights into the study protocol.

Email: nazim@tmsk.uitm.edu.my 